Revue des sciences de l'eau

\title{
Étude de la biosorption du chrome (VI) par une biomasse méditerranéenne : Posidonia oceanica (L.) delile Biosorption of chromium (VI) by a mediterranean biomass: Posidonia oceanica (L.) Delile
}

\section{Mohamed Chaker Ncibi, Borhane Mahjoub et Mongi Seffen}

Volume 21, numéro 4, 2008

Numéro publié dans le cadre de l'Année internationale de l'assainissement

URI : https://id.erudit.org/iderudit/019166ar

DOI : https://doi.org/10.7202/019166ar

Aller au sommaire du numéro

\section{Éditeur(s)}

Université du Québec - INRS-Eau, Terre et Environnement (INRS-ETE)

\section{ISSN}

0992-7158 (imprimé)

1718-8598 (numérique)

\section{Découvrir la revue}

Citer cet article

Ncibi, M. C., Mahjoub, B. \& Seffen, M. (2008). Étude de la biosorption du chrome (VI) par une biomasse méditerranéenne : Posidonia oceanica (L.) delile. Revue des sciences de l'eau / Journal of Water Science, 21(4), 441-449.

https://doi.org/10.7202/019166ar
Résumé de l'article

Les fibres du végétal marin Posidonia oceanica (L.) ont été utilisées en tant que nouvel adsorbant biologique pour l'élimination du chrome hexavalent des solutions aqueuses artificiellement contaminées. Les expériences ont été effectuées en lots aussi bien pour les cinétiques que pour les isothermes d'adsorption. Les variables expérimentales étudiées sont le pH, la température, la quantité de biomasse et la concentration initiale de $\mathrm{Cr}(\mathrm{VI})$. Le maximum de biosorption a été observé pour un $\mathrm{pH}$ 2. La capacité de biosorption semble être optimisée par une augmentation de la température, de la quantité de biosorbant et de la concentration initiale. Les isothermes d'adsorption sont en accord avec les modèles de Redlich-Peterson et Langmuir. De plus, l'analyse thermodynamique a révélé que le présent processus d'adsorption est un phénomène favorable, endothermique et spontané. 


\section{ÉTUDE DE LA BIOSORPTION DU CHROME (VI) PAR UNE BIOMASSE MÉDITERRANÉENNE : POSIDONIA OCEANICA (L.) DELILE}

Biosorption of chromium (VI) by a mediterranean biomass: Posidonia oceanica (L.) Delile

Mohamed Chaker Neibi $^{1,2, *}$, Borhane Mahjoub ${ }^{1}, 2$, Mongi SefFen ${ }^{2}$

${ }^{1}$ Laboratoire de Chimie, Institut Supérieur Agronomique de Chott Meriem, BP 47, Sousse 4042, Tunisie

${ }^{2}$ U.R. Chimie Appliquée et Environnement, École préparatoire aux Académies militaires, Sousse 4000, Tunisie

Reçu le 29 janvier 2007, accepté le 12 mars 2008

\section{RÉSUMÉ}

Les fibres du végétal marin Posidonia oceanica (L.) ont été utilisées en tant que nouvel adsorbant biologique pour l'élimination du chrome hexavalent des solutions aqueuses artificiellement contaminées. Les expériences ont été effectuées en lots aussi bien pour les cinétiques que pour les isothermes d'adsorption. Les variables expérimentales étudiées sont le $\mathrm{pH}$, la température, la quantité de biomasse et la concentration initiale de $\mathrm{Cr}(\mathrm{VI})$. Le maximum de biosorption a été observé pour un $\mathrm{pH}$ 2. La capacité de biosorption semble être optimisée par une augmentation de la température, de la quantité de biosorbant et de la concentration initiale. Les isothermes d'adsorption sont en accord avec les modèles de RedlichPeterson et Langmuir. De plus, l'analyse thermodynamique a révélé que le présent processus d'adsorption est un phénomène favorable, endothermique et spontané.

\section{Mots clés: Biomasse marine; fibres de posidonie; iso- thermes; thermodynamique}

\section{Article publié dans le cadre de l'Année internationale de l'assainissement}

*Auteur pour correspondance :

Téléphone: 216-73-34-85-46

Courriel : ncibi_mo@yahoo.com

\section{SUMMARY}

The marine biomass Posidonia oceanica (L.) fibres were used as a novel low cost biological adsorbent for the removal of hexavalent chromium from artificially contaminated aqueous solutions. Experiments were carried out for sorption kinetics and isotherms in a batch system. The operating variables studied were $\mathrm{pH}$, temperature, biomass quantity and initial chrome concentration. The maximum adsorption removal was observed at $\mathrm{pH} 2$. The biosorption capacity of $P$. oceanica fibres for $\mathrm{Cr}(\mathrm{VI})$ was enhanced by increasing the temperature, the biosorbent amount and initial chromium concentration. The isotherm adsorption data were satisfactorily found to be described by both Redlich-Peterson and Langmuir. Besides, the thermodynamic studies revealed that the present adsorption process is a favourable, endothermic and spontaneous phenomenon.

Keywords: Marine biomass; posidonia fibres; isotherms; thermodynamics 


\section{INTRODUCTION}

Le chrome se présente dans la nature sous plusieurs formes en fonction de son degré d'oxydation. Parmi les deux formes les plus stables (i.e. Cr trivalent et $\mathrm{Cr}$ hexavalent), le $\mathrm{Cr}$ (VI) représente la plus grande menace pour l'environnement et la santé humaine, compte tenu de sa grande toxicité et son potentiel carcinogène (SHANKER et al., 2005). De plus, l'exposition répétée et/ou prolongée à cet élément chimique peut causer des diarrhées, des ulcères et des irritations de la peau et des yeux (TEWARI et al., 2005). Les répercussions nocives sur l'écosystème et sur l'homme, liées au chrome, sont d'autant plus graves si l'on sait que ce métal est le composant majeur de plusieurs filières industrielles, notamment les tanneries et les industries des colorants synthétiques et du cuir (BARNHART, 1997) avec des concentrations variables pouvant atteindre $5 \mathrm{~g} / \mathrm{L}$ de chrome élémentaire selon l'activité industrielle (KRUGER et al., 1997). Face à ce préjudice environnemental, plusieurs procédés de dépollution ont été développés pour éliminer le Cr (VI) des effluents industriels ayant comme objectifs la protection de l'environnement et l'éventuelle réutilisation des eaux, surtout dans les pays arides. Parmi ces techniques, on cite la réduction suivie d'une précipitation chimique(OZER etal., 1997), l'échange ionique(RENGARAG et al., 2003), l'osmose inverse (PADILLA et TAVANI, 1999) et l'adsorption sur charbons actifs commerciaux (LOTFI et ADHOUM, 2002).

Cependant, la plupart de ces technologies sont coûteuses, surtout lorsqu'elles sont appliquées pour les effluents à haut débit. Par conséquent, la technique d'adsorption sur les supports biologiques assez disponible et bon marché peut être à l'origine d'un processus de dépollution techniquement fiable et surtout économiquement viable, surtout que les qualités bioaccumulatrices de la Posidonie vis-à-vis des métaux ont été confirmées (LAFABRIE et al., 2007). Dans ce contexte, plusieurs travaux de recherche ont été menés ces dernières années dans lesquels toute une panoplie d'adsorbants potentiels de $\mathrm{Cr}$ (VI) a été expérimentée. Parmi ces matrices adsorbantes, on cite l'algue verte Spirogyra sp. (GUPTA, 2001), les cônes de pin (UCUN et al., 2002), les semences d'Ocimum basilicum (MELO et D'SOUZA, 2004), des biomasses fongiques (PARK et al., 2005) et les déchets d'Araucaria angustifolia (BRASIL et al., 2006).

L'objectif principal de cette recherche est d'étudier, dans une optique d'optimisation, l'influence de quelques paramètres clés sur la capacité de biosorption du chrome (VI) par une biomasse marine très disponible et bon marché, Posidonia oceanica (L.), et ce, à partir de solutions aqueuses artificiellement polluées. Une modélisation des isothermes d'adsorption et une étude thermodynamique ont été également réalisées afin de comprendre la nature des mécanismes réactionnels mis en jeu lors du présent phénomène de biosorption. Notons que cette biomasse a déjà donné des résultats prometteurs lors d'études d'adsorption du phénol (NCIBI et al., 2006a), de colorants textiles (NCIBI et al., 2006b) et du bleu de méthylène (NCIBI et al., 2007).

\section{MATÉRIELS ET MÉTHODES}

\subsection{Préparation de la biomasse adsorbante}

Les aegagropiles de la phanérogame marine endémique du bassin Méditerranéen, P. oceanica ont été collectées sur la plage de Chott Meriem (côte est de la Tunisie). Les fibres ont été manuellement lavées avec de l'eau distillée afin d'éliminer le sable et les sels. Par la suite, elles ont été séchées dans une étuve à $40^{\circ} \mathrm{C}$ pendant 48 heures jusqu'à ce que le poids des fibres devienne constant, puis conservé dans des dessiccateurs.

\subsection{Préparation de la solution de chrome (VI)}

Une solution mère de $\mathrm{Cr}(\mathrm{VI})$ a été préparée en solubilisant une quantité déterminée de $\mathrm{K}_{2} \mathrm{Cr}_{2} \mathrm{O}_{7}$ dans de l'eau déionisée de façon à obtenir une concentration égale à $100 \mathrm{mg} / \mathrm{L}$, les autres concentrations étant obtenues par des dilutions successives. Le $\mathrm{pH}$ initial de la solution a été ajusté en ajoutant des solutions diluées à $0,1 \mathrm{M}$ de $\mathrm{HCl}$ ou $\mathrm{NaOH}$.

\subsection{Procédure expérimentale}

Les expériences d'adsorption ont été réalisées, en triplicata, en mettant $0,5 \mathrm{~g}$ de fibres en contact avec $50 \mathrm{~mL}$ de la solution de chrome, et ce pour une concentration $(5-50 \mathrm{mg} / \mathrm{L})$ et un $\mathrm{pH}(2-7)$ donnés. Les essais ont été conduits dans un bain-marie thermostaté et agité à 100 oscillations/min. Pour les cinétiques d'adsorption, les échantillons ont été pris à des intervalles de temps prédéterminés, centrifugés pendant $2 \mathrm{~min}$ à 5000 rotation/min puis analysés. Concernant les essais des isothermes, les concentrations initiales utilisées étaient 5; 10; 20; 30; 40; 50 et $60 \mathrm{mg} / \mathrm{L}$. Les prélèvements étaient faits après un temps d'équilibre de 3 heures. La quantité de $\mathrm{Cr}$ (VI) adsorbée par gramme de fibres, $\mathrm{Qa}(\mathrm{mg} / \mathrm{g})$, est calculée selon la formule suivante:

$$
\mathrm{Qa}=(\mathrm{Ci}-\mathrm{Ce}) \frac{\mathrm{V}}{\mathrm{M}}
$$

où $\mathrm{Ci}$ et $\mathrm{Ce}$ sont respectivement les concentrations initiale et finale du chrome en solution qui s'expriment en $\mathrm{mg} / \mathrm{L}$. V est le volume en litre de la solution et $\mathrm{M}$ représente la masse des fibres de $P$. oceanica en gramme. 


\subsection{Analyse du chrome (VI) en solution}

La mesure de la concentration non adsorbée de Cr (VI) a été effectuée selon la méthode colorimétrique standard (GREENBERG et al., 1985). On prélève un échantillon de $1 \mathrm{~mL}$ de la solution qu'on mélange avec un agent complexant, le 1,5-diphenycarbazide en milieu acide. Ainsi, un complexe rose violacé se forme après 10 minutes, et son intensité est mesurée par spectrophotométrie à $540 \mathrm{~nm}$.

\section{RÉSULTATS ET DISCUSSION}

\subsection{Influence du $p H$}

Le $\mathrm{pH}$ initial de la solution est un paramètre important qui doit être pris en considération lors de toute étude d'adsorption. L'effet de ce facteur sur l'évolution de la capacité d'adsorption a été analysé sur une gamme de $\mathrm{pH}$ allant de 2 à 7 . Les résultats présentés sur la figure 1, ont montré que la quantité maximale de $\mathrm{Cr}$ (VI) adsorbée se produit à $\mathrm{pH} 2$ avec $1,64 \mathrm{mg} / \mathrm{g}$, pour une concentrations initiale de $20 \mathrm{mg} / \mathrm{L}$. Cette capacité d'adsorption diminue proportionnellement avec l'augmentation $\mathrm{du} \mathrm{pH}$ pour enregistrer sa plus faible valeur à $\mathrm{pH} 7$ avec $0,07 \mathrm{mg} / \mathrm{g}$. Ce comportement s'explique par le fait qu'à $\mathrm{pH} 2$, les groupements fonctionnels présents à la surface des fibres de posidonie

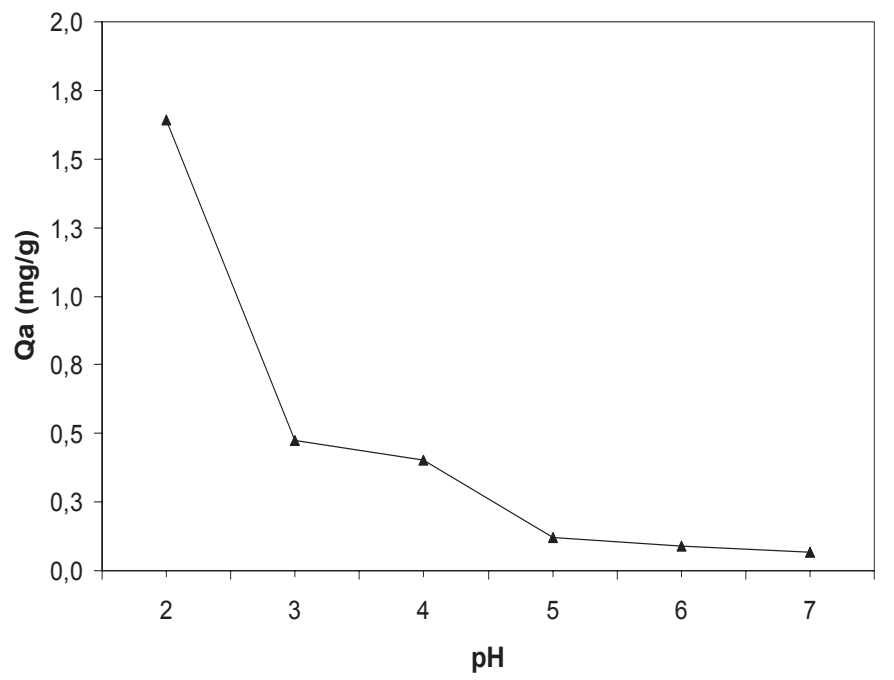

Figure 1. Influence du pH sur la capacité de biosorption du chrome (VI) par les fibres de posidonie (Ratio solide/liquide = $10 \mathrm{~g} / \mathrm{L}$, concentration initiale $=20 \mathrm{mg} / \mathrm{L}$, température $=$ $\left.30 \pm 2^{\circ} \mathrm{C}\right)$.

Effect of $p H$ on the biosorption capacity of chromium (VI) by posidonia fibres (Solid/Liquid ratio $=10 \mathrm{~g} / \mathrm{L}$, initial concentration $=20 \mathrm{mg} / \mathrm{L}$, temperature $=30 \pm 2^{\circ} \mathrm{C}$ ). (i.e. groupements hydroxyles, carboxyles, phosphonates et sulfonates) subissent une forte protonation, ce qui confère aux biopolymères pariétaux une charge globale positive. D'autre part, les formes ioniques du chrome hexavalent qui peuvent être présentes en solution sont de nature anionique telle que $\mathrm{HCrO}_{4}^{-}, \mathrm{Cr}_{2} \mathrm{O}_{7}{ }^{2-}, \mathrm{Cr}_{3} \mathrm{O}_{10}{ }^{2-}$ et $\mathrm{Cr}_{4} \mathrm{O}_{13}{ }^{2-}$ (DÖNMEZ et AKSU, 2002; TEWARI et al., 2005), et ce pour des $\mathrm{pH}$ allant de 1,5 à 4 (UCUN et al., 2002). Ainsi, la biosorption mise en jeu lors de la présente étude semble être essentiellement due à un phénomène d'attraction électrostatique. De plus, la capacité d'adsorption enregistrée diminue avec l'augmentation du $\mathrm{pH}$. En effet, plus le $\mathrm{pH}$ augmente, plus la solution se concentre en radiaux hydroxyles $\left(\mathrm{OH}^{-}\right)$libres qui sont susceptibles de concurrencer les espèces anioniques de $\mathrm{Cr}(\mathrm{VI})$ sur les sites actifs d'adsorption disponible à la surface des fibres de $P$. oceanica. La même tendance vis à vis de l'influence du $\mathrm{pH}$ sur l'adsorption du Cr (VI) a été également signalée pour d'autres matrices biologiques telles que l'algue Dunaliella sp. (DÖNMEZ et AKSU, 2002), les cônes de Pinus sylvestris (UCUN et al., 2002) et le mycélium du champignon Lentinus sajor-caju (BAYRAMOGLU et al., 2005).

\subsection{Influence de la température}

L'étude de l'influence de la température sur l'adsorption du Cr (VI) sur les fibres de P. oceanica, pour une concentration initiale de $20 \mathrm{mg} / \mathrm{L}$ et à un $\mathrm{pH}=2$, est illustrée dans la figure 2. Une nette amélioration de la capacité de biosorption à

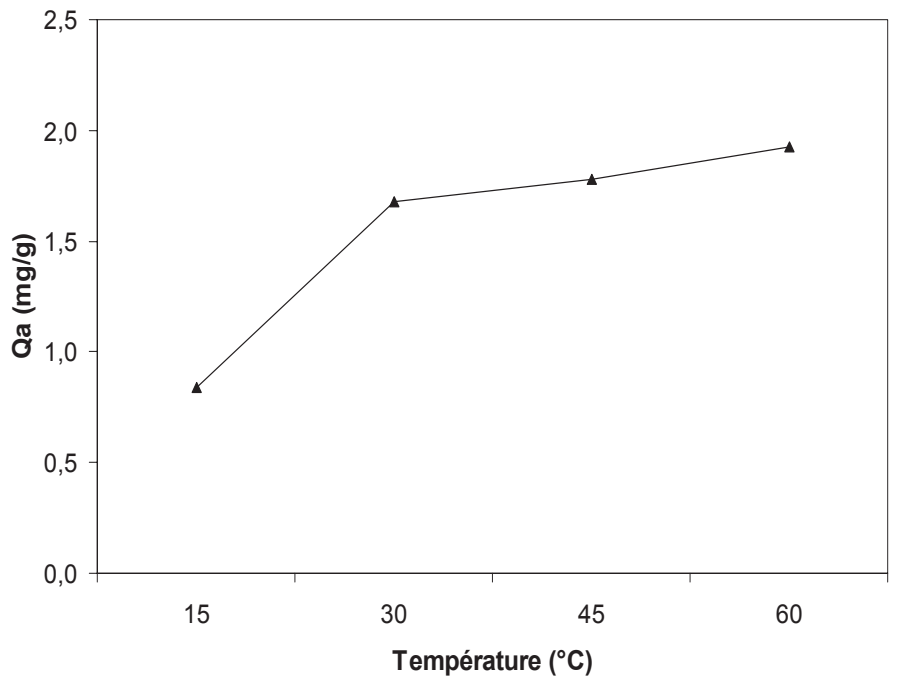

Figure 2 Influence de la température sur la capacité de biosorption du chrome (VI) par les fibres de posidonie (Ratio solide/ liquide $=10 \mathrm{~g} / \mathrm{L}$, concentration initiale $=20 \mathrm{mg} / \mathrm{L}$, $\mathrm{pH}=2$ ).

Effect of temperature on the biosorption capacity of chromium (VI) by posidonia fibres (Solid/Liquid ratio $=$ $10 \mathrm{~g} / \mathrm{L}$, initial concentration $=20 \mathrm{mg} / \mathrm{L}, \mathrm{pH}=2$ ). 
l'équilibre est enregistrée au fur et à mesure que la température augmente, et ce dans les conditions opératoires utilisées. En effet, en augmentant la température de la réaction de 15 à $60^{\circ} \mathrm{C}$, la quantité de chrome adsorbée à l'équilibre a augmenté de 0,94 à $1,82 \mathrm{mg} / \mathrm{g}$. Par conséquent, la biosorption du chrome hexavalent par les fibres brutes de $P$. oceanica semble être un phénomène endothermique. Ce phénomène peut également être dû à une augmentation relative de la mobilité des ions chromates en solution, ce qui améliore leur exposition aux sites actifs d'adsorption d'une part, et les fait parvenir à des sites difficiles d'accès d'autre part. Des résultats similaires ont également été signalés par d'autres équipes de recherche, notamment ARICA et BAYRAMOGLU (2005) et TEWARI et al. (2005). Notons que la nature endothermique du présent phénomène de biosorption sera confirmée ultérieurement lors de l'étude thermodynamique.

\subsection{Influence de la quantité de biomasse}

Des masses de 2, 5, 10, 15 et 20 grammes de fibres de posidonie ont été séparément testées dans un litre de solution de $20 \mathrm{mg} / \mathrm{L}$ de $\mathrm{Cr}(\mathrm{VI})$ à $\mathrm{pH}=2$. La figure 3 montre que la quantité de chrome adsorbée à l'équilibre a augmenté d'une façon significative dans la gamme pondérale examinée. En outre, on observe que le maximum de rétention est obtenu pour une masse de 20 grammes de fibres de P. oceanica par litre de solution. Pour cette quantité, la capacité de biosorption enregistre sa plus haute valeur $(1,92 \mathrm{mg} / \mathrm{g}$; i.e. $93 \%$ de taux de rétention). Pour les autres quantités, la capacité de biosorption a augmenté de 0,39 à $1,78 \mathrm{mg} / \mathrm{g}$ pour les concentrations respectives de fibres de 2 à $20 \mathrm{~g} / \mathrm{L}$ de fibres. Laugmentation du taux de rétention du chrome hexavalent en fonction de l'augmentation de la masse de fibres est principalement due à un accroissement conséquent du nombre de sites actifs d'adsorption à la surface de la biomasse méditerranéenne. Cette même tendance a été rapportée par DAKIKY et al. (2002) qui ont travaillé sur la biosorption de Cr (VI) sur laine, sciure de bois, aiguilles de pin, coques d'amandes et feuilles de cactus.

\subsection{Influence de la concentration initiale}

L'influence de la concentration initiale de chrome (VI) sur la capacité d'adsorption des fibres de $P$. oceanica a été étudiée pour des concentrations de 5,10, 25 et $50 \mathrm{mg}$ de chrome par litre de solution et à $\mathrm{pH}=2$. Les résultats, illustrés dans la figure 4, montrent une augmentation de la quantité de $\mathrm{Cr}$ (VI) adsorbée en fonction de l'augmentation de la concentration initiale. En effet, après un temps d'équilibre de $3 \mathrm{~h}$, la capacité de biosorption enregistre un accroissement de 0,60 à $2,42 \mathrm{mg} / \mathrm{g}$, respectivement pour des concentrations de 5 à $50 \mathrm{mg} / \mathrm{L}$. Ce comportement s'explique par le fait que plus la concentration de chrome augmente, plus le nombre d'ions en solution augmente, impliquant une capacité d'adsorption plus élevée. Le même phénomène a été également signalé par UCUN et al. (2002) et PARK et al. (2005).

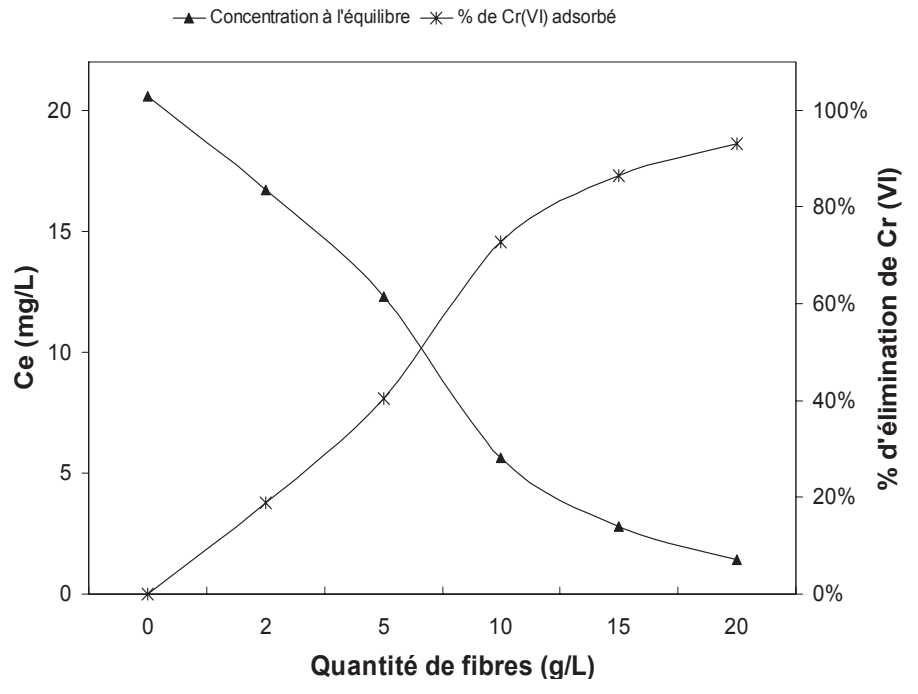

Figure 3. Influence de la quantité de fibres sur la capacité de biosorption du chrome (VI) par les fibres de posidonie (concentration initiale $=20 \mathrm{mg} / \mathrm{L}, \mathrm{pH}=2$, température $=30 \pm 2^{\circ} \mathrm{C}$ ).

Effect of fibres quantity on the biosorption capacity of chromium (VI) by posidonia fibres (initial concentration = $20 \mathrm{mg} / \mathrm{L}, \mathrm{pH}=2$, temperature $=30 \pm 2^{\circ} \mathrm{C}$ ).

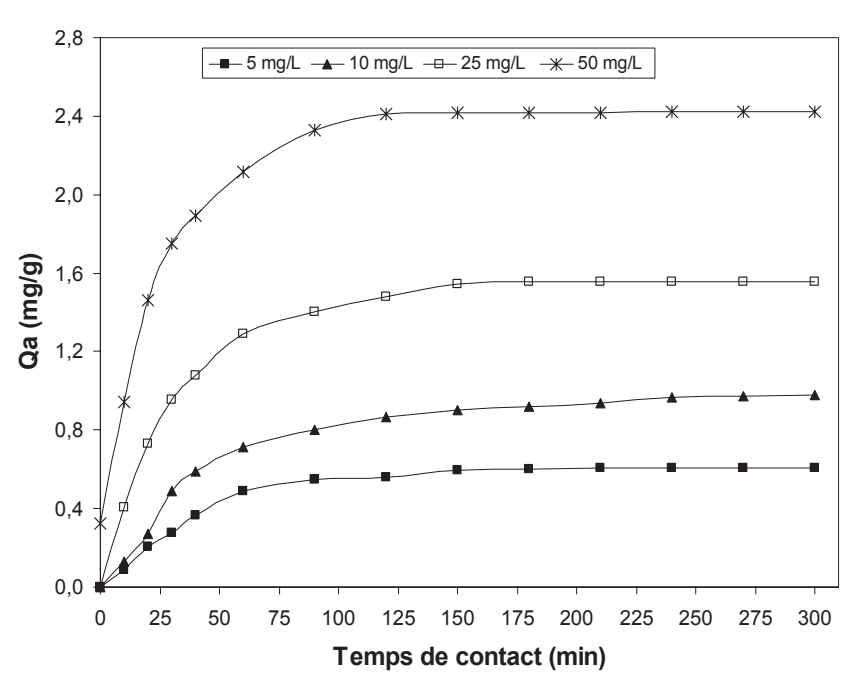

Figure 4. Influence de la concentration initiale sur la capacité de biosorption du chrome (VI) par les fibres de posidonie (Ratio solide/liquide $=10 \mathrm{~g} / \mathrm{L}, \mathrm{pH}=2$, température $=30 \pm 2^{\circ} \mathrm{C}$ ). Influence of initial concentration on the biosorption capacity of chromium (VI) by posidonia fibres (Solid/Liquid ratio $=$ $10 \mathrm{~g} / \mathrm{L}, \mathrm{pH}=2$, temperature $=30 \pm 2^{\circ} \mathrm{C}$ ). 


\subsection{Modélisation des isothermes d'adsorption}

Les courbes expérimentales d'adsorption du présent travail ont été ajustées aux modèles de Langmuir, Freundlich et Redlich-Peterson en utilisant le programme SPSS 13.0. Les formes non linaire et linéaire de l'isotherme de Langmuir (LANGMUIR, 1918) sont respectivement représentées par les équations suivantes:

$$
\begin{aligned}
\mathrm{Q} a & =\frac{\mathrm{Q}^{\circ} \cdot \mathrm{K}_{\mathrm{L}} \cdot \mathrm{Ce}}{1+\mathrm{K}_{\mathrm{L}} \cdot \mathrm{Ce}} \\
\frac{\mathrm{Ce}}{\mathrm{Q} a} & =\frac{1}{\mathrm{~K}_{\mathrm{L}} \cdot \mathrm{Q}^{0}}+\frac{\mathrm{Ce}}{\mathrm{Q}^{0}}
\end{aligned}
$$

où $\mathrm{Q}$ a est la quantité de polluant adsorbée à la surface du support à l'équilibre (en $\mathrm{mg} / \mathrm{g}$ ). $\mathrm{Q}^{\circ}$ représente la quantité maximale d'adsorbat (en $\mathrm{mg} / \mathrm{g}$ ) et $\mathrm{K}_{\mathrm{L}}$ la constante relative à l'énergie d'adsorption (en $\mathrm{L} / \mathrm{g}$ ). La représentation graphique de la variation du rapport $(\mathrm{Ce} / \mathrm{Qa})$ en fonction de (Ce) donne lieu à des droites à partir desquelles les valeurs théoriques $\mathrm{Q}^{\circ}$ et $\mathrm{K}_{\mathrm{L}}$ sont calculées à l'aide des pentes et des ordonnées à l'origine. Les formes non linaire et linéaire de l'isotherme de Freundlich (FREUNDLICH, 1906) sont données par les équations suivantes :

$$
\begin{aligned}
& \mathrm{Qa}=\mathrm{K}_{\mathrm{F}} \cdot(\mathrm{Ce})^{\frac{1}{\mathrm{n}}} \\
& \mathrm{LnQa}=\operatorname{LnK}_{\mathrm{F}}+\frac{1}{\mathrm{n}} \mathrm{LnCe}
\end{aligned}
$$

tel que $\mathrm{K}_{\mathrm{F}}$ est la constante de Freundlich et $\mathrm{n}$ un facteur relatif à l'intensité d'adsorption, appelé aussi facteur d'hétérogénéité. La représentation graphique de la variation de $L_{n} Q_{a}$ en fonction de $\mathrm{L}_{\mathrm{n}} \mathrm{C}_{\mathrm{e}}$ conduit à des droites de régressions à partir desquelles les valeurs théoriques $\mathrm{n}$ et $\mathrm{K}_{\mathrm{F}}$ sont calculées.

Pour le modèle de Redlich-Peterson (JOSSENS et al., 1978), il incorpore les caractéristiques des isothermes de Langmuir et de Freundlich dans une seule équation mathématique (Eq. 6).

$$
\mathrm{Qa}=\left(\mathrm{A}_{\mathrm{R}-\mathrm{P}} \cdot \mathrm{Ce}\right) /\left(1+\mathrm{K}_{\mathrm{R}-\mathrm{P} \cdot \mathrm{Ce}} \mathrm{e}^{\beta}\right)
$$

avec la forme linéaire suivante (Eq. 7) :

$$
\operatorname{Ln}\left(\frac{A_{R-P} \cdot C e}{Q e}-1\right)=\beta \cdot \operatorname{Ln}(C e)+\ln K_{R-P}
$$

où, $A_{R-P}(L / m g) \beta$ et $K_{R-P}(L / g)$ sont des constantes de l'isotherme de Redlich-Peterson, et $\beta$ l'exposant de ce modèle. Notons que le calcul de la constante $A_{R-P}$ nécessite une méthode d'affinement par itération et que l'exposant $\beta$, oscillant entre 0 et 1 , confere au modèle de Redlich-Peterson ses deux tendances limites, à savoir la forme du modèle de Langmuir quand $\beta=1$ et la forme de la loi d'Henry (i.e. le modèle de Freundlich aux basses concentrations et pressions) pour $\beta=0$.

Sur le plan analytique, le degré d'adéquation des modèles théoriques aux résultats expérimentaux a été déterminé en se basant sur les coefficients de détermination $\left(\mathrm{R}^{2}\right)$ d'une part, et le pourcentage de déviation de l'erreur (\% D), d'autre part. Ainsi, les résultats de la modélisation seront considérés comme convenables pour décrire le présent processus de biosorption si la déviation de l'erreur est inférieure à $5 \%$ et le coefficient $\mathrm{R}^{2}$ est supérieur à $95 \%$. Notons que $(\% \mathrm{D})$ est calculé selon la formule suivante :

$$
\% \mathrm{D}=\left(\frac{1}{\mathrm{~N}} \sum_{\mathrm{i}=1}^{\mathrm{N}}\left|\left(\mathrm{Q} \mathrm{e}-\mathrm{Q}_{\text {calc.. }}\right) / \mathrm{Qe}\right|\right) \times 100 \quad(\%)
$$

où, $\mathrm{N}$ est le nombre de points expérimentaux et $\mathrm{Q}_{\text {calc }}$. est la quantité calculée de Cr (VI) adsorbée par unité de masse $(\mathrm{mg} / \mathrm{g})$.

Les résultats relatifs aux constantes des différents modèles, ainsi que les pourcentages de la déviation de l'erreur, sont donnés, pour une température de $30^{\circ} \mathrm{C}$, dans le tableau 1 .

Tableau 1. Constantes de Langmuir, Freundlich et RedlichPeterson relatives à la biosorption de chrome (VI) sur fibres brutes de P. oceanica.

Table 1. Langmuir, Freundlich and Redlich-Peterson isotherms constants related to the biosorption chromium (VI) onto raw P. oceanica.

\begin{tabular}{ll}
\hline$\underline{\text { Langmuir }}$ & \\
\hline $\mathrm{Q}^{\circ}$ (capacité maximale d'adsorption) & 3,34 \\
$\mathrm{~K}_{\mathrm{L}}$ (constante de Langmuir) & 0,14 \\
$\mathrm{R}^{2}$ & 0,997 \\
$\% \mathrm{D}$ (pourcentage de déviation de l'erreur) & 3,36 \\
$\underline{\text { Freundlich }}$ & \\
$\mathrm{K}_{\mathrm{F}}$ (constante de Freundlich) & 0,56 \\
$\mathrm{n}$ (facteur relatif à l'intensité d'adsorption) & 2,06 \\
$\mathrm{R}^{2}$ & 0,969 \\
$\% \mathrm{D}$ & 6,77 \\
$\underline{\text { Redlich-Peterson }}$ & \\
$\mathrm{a}_{\mathrm{R}-\mathrm{P}}$ (constante de Redlich-Peterson) & 0,51 \\
$\mathrm{~K}_{\mathrm{R}-\mathrm{P}}$ (constante de Redlich-Peterson) & 0,18 \\
$\beta$ (exposant de Redlich-Peterson) & 0,97 \\
$\mathrm{R}^{2}$ & 0,997 \\
$\% \mathrm{D}$ & 2,86 \\
\hline
\end{tabular}


L'ajustement des données expérimentales à l'équilibre avec les isothermes de Langmuir, Freundlich et Redlich-Peterson est illustré par la figure 5. Il en sort que les modèles de RedlichPeterson et Langmuir sont les plus appropriés pour décrire le présent phénomène d'adsorption, et ce en se basant sur des coefficients de détermination très élevés ( $99,7 \%$ pour les deux) et des pourcentages de déviation de l'erreur assez faibles $(2,86$ et 3,36 respectivement pour Redlich-Peterson et Langmuir). Concernant le modèle de Freundlich, malgré que le coefficient de détermination est assez élevé $(\approx 0,97)$, la variation de l'erreur calculée s'élève à $6,8 \%$. Ainsi, le modèle de Freundlich ne semble pas être adéquat pour décrire le présent processus de biosorption. De plus, ce résultat de la modélisation est d'autant plus confirmé que l'exposant $\beta$ est très proche de l'unité $(0,97)$, ce qui fait que les équations mathématiques de Redlich-Peterson et de Langmuir sont pratiquement similaires. Par conséquent, les éventuelles hypothèses liées à ce phénomène de biosorption sont celles du modèle de Langmuir, à savoir une adsorption en monocouche sur des sites actifs d'affinités similaires.

De plus, nous avons effectué une étude comparée des capacités d'adsorption de $\mathrm{Cr}$ (VI) entre les fibres de P. oceanica et autres adsorbants potentiels sur la base de la quantité de chrome adsorbée à l'équilibre selon le modèle de Langmuir, tout en tenant compte des conditions expérimentales utilisées. Les résultats de cette comparaison, illustrés dans le tableau 2, ont montré que la biomasse marine, à son état brute, possède d'ors et déjà un potentiel acceptable pour éliminer le Cr (VI)

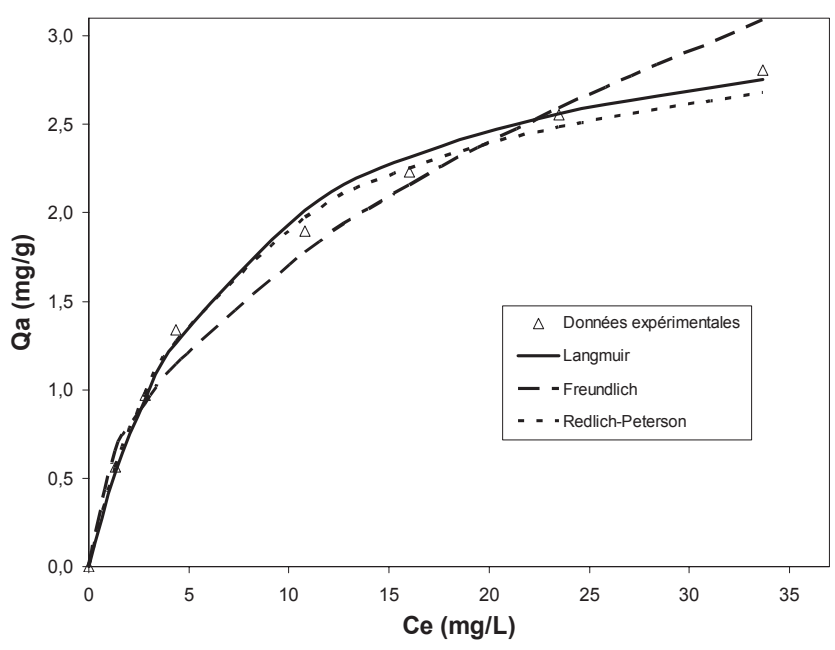

Figure 5. Modélisation de l'isotherme d'adsorption relative à la biosorption de chrome (VI) sur fibres brutes de $P$. oceanica pour une température égale à $30 \pm 2^{\circ} \mathrm{C}$. Adsorption isotherm modelling related to the biosorption of chromium (VI) onto raw P. oceanica for a temperature equal to $30 \pm 2^{\circ} \mathrm{C}$.

Tableau 2 Capacités d'adsorption d'adsorbants non traités vis à vis du chrome (VI) Table 2 Adsorption capacities of non-treated adsorbents toward chromium (VI)

\begin{tabular}{|c|c|c|c|c|}
\hline Adsorbants & $\begin{array}{l}\text { Température } \\
\left({ }^{\circ} \mathrm{C}\right)\end{array}$ & $\mathrm{pH}$ & $\begin{array}{c}\text { Capacité d'adsorption } \\
\mathrm{Q}^{\circ}(\mathrm{mg} / \mathrm{g})\end{array}$ & Références \\
\hline Fibres de $P$. oceanica & 30 & 2 & 3,34 & cette étude \\
\hline Feuilles de cactus & 30 & 2 & 7,08 & DAKIKY et al., 2002 \\
\hline Charbon actif commercial & 20 & 4 & 4,72 & $\begin{array}{c}\text { BABEL et KURNIAWAN, } \\
2004\end{array}$ \\
\hline Tiges d'Atriplex canescens & 25 & 5 & 3,4 & SAWALHA et al., 2005 \\
\hline Feuilles d'agaves & 22 & 2 & 3,3 & GARDEA et al., 2000 \\
\hline Sciures pyrolisées & 30 & 2 & 2,2 & HAMADI et al., 2001 \\
\hline $\begin{array}{l}\text { Charbon issu des coques de noix de } \\
\text { coco }\end{array}$ & 22 & 6 & 2,18 & $\begin{array}{l}\text { BABEL et KURNIAWAN, } \\
2004\end{array}$ \\
\hline Bentonite & 25 & 2 & 0,57 & KHAN et al., 1995 \\
\hline Neurospora crassa & 25 & 1 & 0,43 & TUNALI et al., 2005 \\
\hline Croûte de soja & 28 & 1 & 0,28 & DANESHVAR et al., 2002 \\
\hline
\end{tabular}


des effluents aqueux. En effet, par rapport à une sciure pyrolisée, une argile (Bentonite) et même un charbon issu des coques de noix de coco, les fibres de posidonie possèdent une meilleure capacité d'adsorption. Cependant, vis à vis d'autres matériaux, cette même capacité semble être assez basse. Ainsi, pour pouvoir concurrencer ces adsorbants encore plus performants, des prétraitements chimiques ainsi que l'immobilisation de microorganismes (bactéries ou champignons) peuvent être envisagés.

\subsection{Etude thermodynamique}

Les paramètres thermodynamiques ont été déterminés pour pouvoir qualifier le phénomène de biosorption du $\mathrm{Cr}$ (VI) sur fibres de posidonie. Ainsi, l'énergie libre de Gibbs ou enthalpie libre d'adsorption; $\Delta \mathrm{G}^{\circ}(\mathrm{kJ} / \mathrm{mol})$, l'enthalpie d'adsorption; $\Delta \mathrm{H}^{\circ}(\mathrm{kJ} / \mathrm{mol})$ et l'entropie d'adsorption; $\Delta S^{\circ}(\mathrm{J} / \mathrm{mol} / \mathrm{K})$ ont été calculés selon les équations $(9,10)$.

$$
\begin{aligned}
& \Delta \mathrm{G}^{0}=-\mathrm{RT} \cdot \operatorname{Ln}(\mathrm{Kd}) \\
& \operatorname{Ln}(\mathrm{Kd})=\frac{\Delta \mathrm{H}^{0}}{\mathrm{RT}}+\frac{\Delta \mathrm{S}^{0}}{\mathrm{R}}
\end{aligned}
$$

où, $\mathrm{Kd}$ est la constante d'équilibre d'adsorption déduite à partir de la pente du graphe $\operatorname{Ln}(\mathrm{Qa})$ en fonction de $\mathrm{Ce}$ (SAWALHA et al., 2005), R est la constante des gaz parfaits et $\mathrm{T}$ la température en ${ }^{\circ} \mathrm{Kelvin}$. D'après les résultats illustrés dans le tableau 3, la valeur négative de $\left(\Delta S^{\circ}\right)$ indique la nature favorable du présent phénomène d'adsorption. D'autre part, les valeurs négatives de $\left(\Delta \mathrm{G}^{\circ}\right)$ montrent que le processus d'adsorption étudié est spontané jusqu'à une température de $45^{\circ} \mathrm{C}$. Également, la valeur positive de $\left(\Delta \mathrm{H}^{\circ}\right)$ prouve la nature endothermique de ce phénomène (SMITH et VAN NESS, 1987).

Tableau 3. Paramètres thermodynamiques relatives à la biosorption de chrome (VI) sur fibres brutes de P. oceanica.

Table 3. Thermodynamic parameters related to the biosorption chromium (VI) onto raw P. oceanica.

\begin{tabular}{ccccccc}
\hline & \multicolumn{2}{c}{ Enthalpie libre, $\Delta \mathbf{G}^{\circ}(\mathbf{k J} / \mathbf{m o l})$} & $\begin{array}{c}\text { Enthalpie, } \\
\Delta \mathbf{H}^{\circ}\end{array}$ & $\begin{array}{c}\text { Entropie, } \\
\Delta \mathbf{S}^{\circ}\end{array}$ \\
\hline & $15^{\circ} \mathrm{C}$ & $30^{\circ} \mathrm{C}$ & $45^{\circ} \mathrm{C}$ & $60^{\circ} \mathrm{C}$ & $(\mathrm{kJ} / \mathrm{mol})$ & $(\mathrm{J} / \mathrm{mol} / \mathrm{K})$ \\
$\mathrm{Cr}(\mathrm{VI})$ & $-6,29$ & $-1,68$ & $-0,63$ & 2,31 & 241,36 & $-756,22$ \\
\hline
\end{tabular}

\section{CONCLUSIONS}

Dans le présent travail de recherche, nous avons étudié la capacité de biosorption du chrome hexavalent par des fibres d'un végétal marin très disponible sur les côtes méditerranéennes, Posidonia oceanica (L.). Il a été démontré que la capacité d'adsorption à l'équilibre peut être optimisée en augmentant la quantité de fibres, en élevant la température et en augmentant la concentration initiale de chrome, dans les intervalles expérimentés. La modélisation des isothermes a révélé que les modèles de Redlich-Peterson et Langmuir décrivent d'une manière satisfaisante le présent processus d'adsorption. Pour les paramètres thermodynamiques, l'analyse des résultats a montré que l'adsorption du Cr (VI) sur fibres de posidonie est un phénomène spontané, endothermique et favorable.

Par conséquent, compte tenu de l'ensemble des résultats fournis par cette étude, les fibres bon marché et assez disponible de $P$. oceanica pourraient être considérés comme un matériau biologique assez prometteur pour être utilisé en tant qu'adsorbant efficace pour l'élimination du chrome (VI) présent dans les effluents liquides.

\section{REMERCIEMENTS}

Les auteurs remercient l'Agence Universitaire de la Francophonie (AUF) pour la bourse doctorale octroyée au premier auteur, ainsi que les ministères Tunisiens de la Défense Nationale et de la Recherche Scientifique, Technologies et Développement des Compétences pour leurs assistances technique et financière.

\section{RÉFÉRENCES BIBLIOGRAPHIQUES}

ARICA, M.Y., G. BAYRAMOGLU (2005). Cr (VI) biosorption from aqueous solutions using free and immobilized biomass of Lentinus sajor-caju: preparation and kinetic characterization. Colloids and Surfaces A: Physicochem. Eng. Aspects, 253, 203-211.

BABEL, S., T.A. KURNIAWAN (2004). Cr(VI) removal from synthetic wastewater using coconut shell charcoal and commercial activated carbon modified with oxidizing agents and/or chitosan. Chemosphere, 54, 951-967.

BARNHART, J. (1997). Occurrences, uses, and properties of chromium. Regul. Toxicol. Pharmacol, 26, 3-7. 
BAYRAMOGLU, G., G. ELIK, E. YALCIN, M. YILMAZ, M.Y. ARICA, 2005. Modification of surface properties of Lentinus sajor-caju mycelia by physical and chemical methods: evaluation of their Cr (VI) removal efficiencies from aqueous medium. J. Hazardous Mater., B119, 219229.

BRASIL, J.L., R.R. EV, C.D. MILCHAREK, L.C.MARTINS, F.A. PAVAN, A.A. DOS SANTOS, L.P. DIAS, J. DUPONT, C.P. ZAPATA, NORENA, E.C. LIMA (2006). Statistical design of experiments as a tool for optimizing the batch conditions to $\mathrm{Cr}(\mathrm{VI})$ biosorption on Araucaria angustifolia wastes. J. Hazardous Mater., B133, 143-153.

DAKIKY, M., M. KHAMIS, A. MANASSRA, M. MEREB (2002). Selective adsorption of chromium (VI) in industrial wastewater using low-cost abundantly available adsorbents. Adv. Environ. Res., 6, 533-540.

DANESHVAR, N., D. SALARI, S. ABER (2002). Chromium adsorption and $\mathrm{Cr}(\mathrm{VI})$ reduction to trivalent chromium in aqueous solutions by soya cake. J. Hazardous Mater., B94, 49-61.

DÖNMEZ, G., Z. AKSU (2002). Removal of chromium (VI) from saline wastewaters by Dunaliella species. Process Biochem., 38, 751-762

FREUNDLICH, H., 1906. Over the adsorption in solution. J. Phys. Chem., 57, 385-470.

GARDEA, J.L., K.J. TIEMANN, V. ARMENDARIZ, L. BESS, R.R. CHIANELLI, J. RIOS, J.G. PARSONS, G. GAMEZ (2000). Characterization of Cr(VI) binding and reduction to $\mathrm{Cr}$ (III) by the agricultural by products of Avena monida (oat) biomass, J. Hazardous. Mater., 80, 175-181.

GREENBERG, A.E., R.R. TRUSSELL, L.S. CLESCERY, (1985). Standard methods for the examination of water and wastewater. (APHA), 16e edition; AWWA-WPCF, Washington, DC.

GUPTA, V.K., A.K. SHRIVASTAVA, J. NEERAJ (2001). Biosorption of chromium (VI) from aqueous solutions by green algae Spirogyra species. Water Res., 35, 4079-4085.

HAMADI, N.K., X.D. CHEN, M.M. FARID, M.G.Q. LU, (2001). Adsorption kinetics for the removal of chromium (VI) from aqueous solution by adsorbents derived from used tyres and sawdust. Chem. Eng. J., 84, 95-105.
JOSSENS,L.,J.M.PRAUSNIZ,W.FRITZ,U. SCHLUNDER,

A.L. MYERS (1978). Thermodynamics of multisolute adsorption from dilute aqueous solutions. Chem. Eng. Sci., 33, 1097-1106.

KHAN, S.A., R. REHMAN, M.A. KHAN (1995). Adsorption of chromium (III), chromium (VI), and silver (I) on bentonite. Waste Manage., 15, 271-282.

KRUGER, L., T. ANDERSON, J. COATS (1997). Phytoremediation of Soil and Water Contaminants. ACS Symposium Series. Washington DC, American Chem.Soc., 664, 274-282.

LAFABRIE, C., C. PERGENT-MARTINI, G. PERGENT (2007). Metal contamination of Posidonia oceanica meadows along the Corsican coastline (Mediterranean). Environ. Pollut., doi:10.1016/j.envpol.2007.01.047, Sous Presse.

LANGMUIR, I., 1918. The adsorption of gases on plane surfaces of glass, mica and platinum. J. Amer. Chem. Soc., 40, 1361-1403.

LOTFI, M. et N. ADHOUM (2002). Modified activated carbon for the removal of copper, zinc, chromium and cyanide from wastewater. Sep. Purif. Technol., 137-146.

MELO, J.S. et S.F. D'SOUZA (2004). Removal of chromium by mucilaginous seeds of Ocimum basilicum. Bioresour. Technol., 92, 151-155.

NCIBI, M.C., B. MAHJOUB et M. SEFFEN (2006a). Biosorption of phenol onto Posidonia oceanica (L.) seagrass in batch system: Equilibrium and kinetic modelling. Cnd. J. Chem. Eng., 84, 495-500.

NCIBI, M.C., B. MAHJOUB et M. SEFFEN (2006b) Studies on the biosorption of textile dyes from aqueous solutions using Posidonia oceanica (L.) leaf sheaths fibres. Adsorpt. Sci. Technol., 24 (6), 461-473.

NCIBI, M.C., B. MAHJOUB et M. SEFFEN (2007). Kinetic and equilibrium studies of methylene blue biosorption by Posidonia oceanica (L.) fibres. J. Hazard. Mater., B139, 280-285.

OZER, A., H.S. ALTUNDOGAN, M. ERDEM et F. TUMEN (1997). A study on the $\mathrm{Cr}(\mathrm{VI})$ removal from aqueous solutions by steel wool. Environ. Pollut., 97, 107-112.

PADILLA, A.P. et E.L. TAVANI (1999). Treatment of an industrial effluent by reverse osmosis. Desalination, 129, 219-226. 
PARK, D., Y.S. YUN et J.M. PARK (2005). Use of dead fungal biomass for the detoxification of hexavalent chromium: screening and kinetics. Process Biochem., 40, 2559-2565.

RENGARAJ, S., C.K. JOO, Y. KIM et J. YI (2003). Kinetics of removal of chromium from water and electronic process wastewater by ion exchange resins: $1200 \mathrm{H}, 1500 \mathrm{H}$ and IRN97H. J. Hazard. Mater., 102, 257-275.

SAWALHA, M.F., J.L. GARDEA-TORRESDEY, J.G. PARSONS, G. SAUPE et J.R. PERALTA-VIDEA (2005). Determination of adsorption and speciation of chromium species by saltbush (Atriplex canescens) biomass using a combination of XAS and ICP-OES. Microchem. J., 81, 122-132.

SHANKER, A., C. CERVANTES, H. LOZA-TAVERA et S. AVUDAINAYAGAM (2005). Chromium toxicity in plants. Environ. Int., 31, 739-753.

SMITH, J.M. et H.C. VAN NESS (1987). Introduction to chemical engineering thermodynamics, $4 \mathrm{e}$ edition, McgGrawHill, Singapore.

TEWARI, N., P. VASUDEVAN et B. GUHA (2005). Study on biosorption of $\mathrm{Cr}(\mathrm{VI})$ by Mucor hiemalis. Biochem. Eng. J., 23, 185-192.

TUNALI, S., I. KIRAN et T. AKAR (2005). Chromium (VI) biosorption characteristics of Neurospora crassa fungal biomass. Minerals Eng., 18, 681-689.

UCUN, H., Y.K. BAYHAN, Y. KAYA, A. CAKICI et O.F. ALGUR (2002). Biosorption of chromium(VI) from aqueous solution by cone biomass of Pinus sylvestris. Bioresour. Technol., 85, 155-158. 